\title{
Ground-State Analysis for an Exactly Solvable Coupled-Spin Hamiltonian
}

Eduardo MATTEI ${ }^{\dagger}$ and Jon LINKS $\ddagger$

$\dagger$ Centro Brasileiro de Pesquisas Físicas, Rua Dr. Xavier Sigaud 150, Rio de Janeiro, Brazil E-mail: eduardo.mattei@ufrgs.br

$\ddagger$ School of Mathematics and Physics, The University of Queensland, 4072, Australia E-mail: jrl@maths.uq.edu.au

Received July 23, 2013, in final form November 22, 2013; Published online November 30, 2013 http://dx.doi.org/10.3842/SIGMA.2013.076

\begin{abstract}
We introduce a Hamiltonian for two interacting $\mathfrak{s u}(2)$ spins. We use a meanfield analysis and exact Bethe ansatz results to investigate the ground-state properties of the system in the classical limit, defined as the limit of infinite spin (or highest weight). Complementary insights are provided through investigation of the energy gap, ground-state fidelity, and ground-state entanglement, which are numerically computed for particular parameter values. Despite the simplicity of the model, a rich array of ground-state features are uncovered. Finally, we discuss how this model may be seen as an analogue of the exactly solvable $p+i p$ pairing Hamiltonian.
\end{abstract}

Key words: mean-field analysis; Bethe ansatz; quantum phase transition

2010 Mathematics Subject Classification: 81R05; 17B80; 81R12

\section{Introduction}

We will analyse a Hamiltonian for two interacting $\mathfrak{s u}(2)$ spins, and begin by defining the model. The $\mathfrak{s u}(2)$ algebra has generators $\left\{S^{z}, S^{+}, S^{-}\right\}$with commutation relations

$$
\left[S^{+}, S^{-}\right]=2 S^{z}, \quad\left[S^{z}, S^{ \pm}\right]= \pm S^{ \pm}
$$

Given a finite-dimensional module with highest weight $s(2 s \in \mathbb{N} \cup\{0\})$ the action of the generators on the weight basis is

$$
\begin{aligned}
S^{z}|s, m\rangle & =m|s, m\rangle, \\
S^{+}|s, m\rangle & =\sqrt{(s-m)(s+m+1)}|s, m+1\rangle, \\
S^{-}|s, m\rangle & =\sqrt{(s+m)(s-m+1)}|s, m-1\rangle
\end{aligned}
$$

with $m=-s,-s+1, \ldots, s-1, s$.

We will study the following coupled-spin Hamiltonian

$$
H=(1+G)\left(z_{1}^{2}\left(S_{1}^{z}+s\right)+z_{2}^{2}\left(S_{2}^{z}+s\right)\right)-G\left(z_{1} S_{1}^{+}+z_{2} S_{2}^{+}\right)\left(z_{1} S_{1}^{-}+z_{2} S_{2}^{-}\right),
$$

which acts on the $(2 s+1)^{2}$-dimensional tensor product space with basis

$$
\left\{\left|s, m_{1}\right\rangle \otimes\left|s, m_{2}\right\rangle: m_{j}=-s,-s+1, \ldots, s-1, s ; j=1,2\right\} .
$$

The Hamiltonian commutes with $S^{z}=S_{1}^{z}+S_{2}^{z}$. Throughout we assume $z_{1}>z_{2} \geq 0$. For given $s$ the model has three coupling parameters. One of these can be fixed by choosing the overall energy scale, e.g., setting $z_{1}=1$, which leaves two independent parameters $z_{2}$ and $G$. Our main 
objective is to investigate ground-state properties of the model in the classical limit which we define by a sequence of ordered triples $(G, s, m)$ such that $G \rightarrow 0, s \rightarrow \infty, m \rightarrow \pm \infty$ (or $m=0$ ) with $\mu=m /(2 s)$ and $g=4 s G$.

An advantage of this model is that analytic results are accessible. Despite the simplicity of the Hamiltonian, a rich array of ground-state features are uncovered. In Section 2 we conduct an analysis of the system using a mean-field approximation. In contrast, Section 3 is devoted to the exact calculation of certain properties, including results obtained from an exact Bethe ansatz solution of the Hamiltonian, in order to obtain the ground-state phase diagram. In Section 4 we numerically analyse the system to study the ground-state energy gap, fidelity [21, 22], and entanglement $[12,13]$, which have been used as indicators of quantum phase transitions in other simple bipartite quantum systems, e.g., [6, 14, 17]. Conclusions are drawn in Section 5.

The model considered here may be viewed as a spin-analogue of the exactly solvable $p+i p$ pairing Hamiltonian $[4,7,16]$. The Hamiltonian came to some prominence through the work of Volovik [20] and Read and Green [15] who exposed a change in the topological properties of the ground-state wavefunction of the Hamiltonian as a function of the coupling parameter. This result has generated many subsequent studies, as the topological nature of the transition is quite distinctive from conventional forms of phase transition characterised by an order parameter. In the Conclusion we will provide further discussion on this point and the relation between the coupled-spin model and the $p+i p$ model.

We also remark that a related study was recently given in [9] in connection with the LipkinMeshkov-Glick model. A significant difference however is that [9] realises the Hamiltonian with infinite-dimensional unitary representations of coupled $\mathfrak{s u}(1,1)$ spins, in contrast to our approach using finite-dimensional unitary representations of the coupled $\mathfrak{s u}(2)$ spins.

\section{Mean-field analysis}

The Hamiltonian (2) can be approximated by a Hamiltonian with linear terms through

$$
A B \approx A\langle B\rangle+\langle A\rangle B-\langle A\rangle\langle B\rangle
$$

where $\langle A\rangle$ denotes the expectation value of the operator $A$. The mean-field approximation which linearises the Hamiltonian (2) is

$$
\begin{aligned}
H_{\mathrm{mf}}= & (1+G) s\left(z_{1}^{2}+z_{2}^{2}\right)+\rho m+\left((1+G) z_{1}^{2}-\rho\right) S_{1}^{z}+\left((1+G) z_{2}^{2}-\rho\right) S_{2}^{z} \\
& -\frac{\hat{\Delta}}{2}\left(z_{1} S_{1}^{+}+z_{2} S_{2}^{+}\right)-\frac{\hat{\Delta}^{*}}{2}\left(z_{1} S_{1}^{-}+z_{2} S_{2}^{-}\right)+\frac{\Delta^{2}}{4 G},
\end{aligned}
$$

where $\rho$ is a Lagrange multiplier, which is introduced since the mean-field approximation breaks the conservation of $S^{z}$, and

$$
\hat{\Delta}=2 G\left\langle z_{1} S_{1}^{-}+z_{2} S_{2}^{-}\right\rangle .
$$

Setting $\Delta=|\hat{\Delta}|$ the ground state energy is found to be

$$
\begin{aligned}
E_{0}= & (1+G) s\left(z_{1}^{2}+z_{2}^{2}\right)+\rho m+\frac{\Delta^{2}}{4 G}-s \sqrt{\left((1+G) z_{1}^{2}-\rho\right)^{2}+z_{1}^{2} \Delta^{2}} \\
& -s \sqrt{\left((1+G) z_{2}^{2}-\rho\right)^{2}+z_{2}^{2} \Delta^{2}} .
\end{aligned}
$$

Using the Hellmann-Feynman theorem, we can determine mean-field expectation values:

$$
\left\langle\frac{\partial H_{\mathrm{mf}}}{\partial G}\right\rangle=\frac{\partial E_{0}}{\partial G}
$$


which leads to

$$
z_{1}^{2}\left\langle S_{1}^{z}\right\rangle+z_{2}^{2}\left\langle S_{2}^{z}\right\rangle+\frac{\Delta^{2}}{2 G^{2}}=s z_{1}^{2} \frac{(1+G) z_{1}^{2}-\rho+\Delta^{2} G^{-1}}{\sqrt{\left((1+G) z_{1}^{2}-\rho\right)^{2}+z_{1}^{2} \Delta^{2}}}+s z_{2}^{2} \frac{(1+G) z_{2}^{2}-\rho+\Delta^{2} G^{-1}}{\sqrt{\left((1+G) z_{2}^{2}-\rho\right)^{2}+z_{2}^{2} \Delta^{2}}} .
$$

Taking the classical limit gives

$$
\frac{2}{g}=\frac{z_{1}^{2}}{\sqrt{\left(z_{1}^{2}-\rho\right)^{2}+z_{1}^{2} \Delta^{2}}}+\frac{z_{2}^{2}}{\sqrt{\left(z_{2}^{2}-\rho\right)^{2}+z_{2}^{2} \Delta^{2}}} .
$$

Also

$$
\left\langle\frac{\partial H_{\mathrm{mf}}}{\partial \rho}\right\rangle=\frac{\partial E_{0}}{\partial \rho}
$$

yielding

$$
-\left\langle S_{1}^{z}\right\rangle-\left\langle S_{2}^{z}\right\rangle=\frac{s\left((1+G) z_{1}^{2}-\rho\right)}{\sqrt{\left((1+G) z_{1}^{2}-\rho\right)^{2}+z_{1}^{2} \Delta^{2}}}+\frac{s\left((1+G) z_{2}^{2}-\rho\right)}{\sqrt{\left((1+G) z_{2}^{2}-\rho\right)^{2}+z_{2}^{2} \Delta^{2}}} .
$$

Taking the classical limit and using (4) gives

$$
\frac{2}{g}+2 \mu=\frac{\rho}{\sqrt{\left(z_{1}^{2}-\rho\right)^{2}+z_{1}^{2} \Delta^{2}}}+\frac{\rho}{\sqrt{\left(z_{2}^{2}-\rho\right)^{2}+z_{2}^{2} \Delta^{2}}} .
$$

Returning to the energy expression in the limit $s \rightarrow \infty$, and taking into account equations (4), (5), we obtain the intensive energy expression

$$
\begin{aligned}
e_{0} & =\lim _{s \rightarrow \infty} \frac{E_{0}}{s}=\left(z_{1}^{2}+z_{2}^{2}\right)+2 \rho \mu+\frac{\Delta^{2}}{g}-\sqrt{\left(z_{1}^{2}-\rho\right)^{2}+z_{1}^{2} \Delta^{2}}-\sqrt{\left(z_{2}^{2}-\rho\right)^{2}+z_{2}^{2} \Delta^{2}} \\
& =z_{1}^{2}\left(1-\frac{1}{2} \frac{2 z_{1}^{2}+\Delta^{2}-2 \rho}{\sqrt{\left(z_{1}^{2}-\rho\right)^{2}+z_{1}^{2} \Delta^{2}}}\right)+z_{2}^{2}\left(1-\frac{1}{2} \frac{2 z_{2}^{2}+\Delta^{2}-2 \rho}{\sqrt{\left(z_{2}^{2}-\rho\right)^{2}+z_{2}^{2} \Delta^{2}}}\right) .
\end{aligned}
$$

It may be verified that setting $\rho=\Delta^{2} / 4$ in (4), (5) and combining these equations leads to

$$
\mu=1-2 g^{-1} .
$$

Furthermore, setting $\rho=\Delta^{2} / 4$ in (6) gives $e_{0}=0$.

Recalling the assumption $z_{1}>z_{2}$ the minimum excitation energy (i.e. the gap) is

$$
\Delta E=\sqrt{\left(z_{2}^{2}-\rho\right)^{2}+z_{2}^{2} \Delta^{2}},
$$

which vanishes when $z_{2}=\rho=0$. However turning to (5) we see that the limits $z_{2} \rightarrow 0$ and $\rho \rightarrow 0$ do not commute in that equation. First setting $\rho=0$ leads to

$$
\mu=-g^{-1} \text {. }
$$

Alternatively first setting $z_{2}=0$ yields

$$
\mu=\frac{1}{2} \lim _{\rho \rightarrow 0} \frac{\rho}{|\rho|}-\frac{1}{g},
$$

which shows a discontinuity as $\rho$ passes through zero. This indicates that while gapless excitations occur in the limit $z_{2}, \rho \rightarrow 0$, where they are to be found in the phase diagram will depend on how these two limits are taken (at least within the mean-field approximation). A unusual property is that equations (7), (9), (10) do not depend on $z_{1}$ and $z_{2}$. To gain a better appreciation of these features we next conduct an analysis using exact results. 


\section{The exact solution}

We start with the observation that

$$
H(|s,-s\rangle \otimes|s,-s\rangle)=0 .
$$

To determine exact eigenstates of (2) by way of a Bethe ansatz solution, we define generic states of the form

$$
\begin{aligned}
& |\psi\rangle=\prod_{k=1}^{M} C\left(y_{k}\right)(|s,-s\rangle \otimes|s,-s\rangle), \\
& \left|\psi_{j}\right\rangle=\prod_{k \neq j}^{M} C\left(y_{k}\right)(|s,-s\rangle \otimes|s,-s\rangle), \\
& \left|\psi_{j l}\right\rangle=\prod_{k \neq j, l}^{M} C\left(y_{k}\right)(|s,-s\rangle \otimes|s,-s\rangle),
\end{aligned}
$$

where

$$
C(y)=\frac{z_{1} S_{1}^{+}}{y-z_{1}^{2}}+\frac{z_{2} S_{2}^{+}}{y-z_{2}^{2}} .
$$

We also define

$$
H_{0}=z_{1}^{2}\left(S_{1}^{z}+s\right)+z_{2}^{2}\left(S_{2}^{z}+s\right), \quad Q^{+}=z_{1} S_{1}^{+}+z_{2} S_{2}^{+}, \quad Q^{-}=z_{1} S_{1}^{-}+z_{2} S_{2}^{-},
$$

such that

$$
H=(1+G) H_{0}-G Q^{+} Q^{-},
$$

and note the following commutation relations:

$$
\begin{aligned}
& {\left[H_{0}, C(y)\right]=\frac{z_{1}^{3}}{y-z_{1}^{2}} S_{1}^{+}+\frac{z_{2}^{3}}{y-z_{2}^{2}} S_{2}^{+}=y C(y)-Q^{+},} \\
& {\left[Q^{-}, C(y)\right]=-2 \frac{z_{1}^{2}}{y-z_{1}^{2}} S_{1}^{z}-2 \frac{z_{2}^{2}}{y-z_{2}^{2}} S_{2}^{z} .}
\end{aligned}
$$

By direct calculation it is then found that

$$
\begin{aligned}
H|\Psi\rangle= & (1+G) H_{0}|\Psi\rangle-G Q^{+} Q^{-}|\Psi\rangle=(1+G) \sum_{j=1}^{M}\left(y_{j}|\Psi\rangle-Q^{+}\left|\Psi_{j}\right\rangle\right) \\
& +2 G Q^{+} \sum_{j=1}^{M} \sum_{p=1}^{2}\left(C\left(y_{1}\right) \cdots\left(\frac{z_{p}^{2}}{y-z_{p}^{2}} S_{p}^{z}\right) \cdots C\left(y_{M}\right)\right)|0\rangle \\
= & (1+G) \sum_{j=1}^{M}\left(y_{j}|\Psi\rangle-Q^{+}\left|\Psi_{j}\right\rangle\right)+2 G Q^{+} \sum_{j=1}^{M}\left(\frac{z_{1}^{2} s}{y_{j}-z_{1}^{2}}+\frac{z_{2}^{2} s}{y_{j}-z_{2}^{2}}\right)\left|\Psi_{j}\right\rangle \\
& +2 G Q^{+} \sum_{j=1}^{M} \sum_{r>j}^{M} \sum_{p=1}^{2} \frac{z_{p}^{3}}{\left(y_{j}-z_{p}^{2}\right)\left(y_{r}-z_{p}^{2}\right)} S_{p}^{+}\left|\Psi_{r j}\right\rangle \\
= & (1+G) \sum_{j=1}^{M}\left(y_{j}|\Psi\rangle-Q^{+}\left|\Psi_{j}\right\rangle\right)-2 G Q^{+} \sum_{j=1}^{M}\left(\frac{z_{1}^{2} s}{y_{j}-z_{1}^{2}}+\frac{z_{2}^{2} s}{y_{j}-z_{2}^{2}}\right)\left|\Psi_{j}\right\rangle
\end{aligned}
$$




$$
\begin{aligned}
& +G Q^{+} \sum_{j=1}^{M} \sum_{r \neq j}^{M} \sum_{p=1}^{2}\left(\frac{z_{p} y_{r}}{\left(y_{j}-y_{r}\right)\left(y_{r}-z_{p}^{2}\right)}+\frac{z_{p} y_{j}}{\left(y_{r}-y_{j}\right)\left(y_{j}-z_{p}^{2}\right)}\right) S_{p}^{+}\left|\Psi_{r j}\right\rangle \\
= & (1+G) \sum_{j=1}^{M}\left(y_{j}|\Psi\rangle-Q^{+}\left|\Psi_{j}\right\rangle\right)-2 G Q^{+} \sum_{j=1}^{M}\left(\frac{z_{1}^{2} s}{y_{j}-z_{1}^{2}}+\frac{z_{2}^{2} s}{y_{j}-z_{2}^{2}}\right)\left|\Psi_{j}\right\rangle \\
& +G Q^{+} \sum_{j=1}^{M} \sum_{r \neq j}^{M}\left(\frac{y_{r}}{y_{j}-y_{r}}\left|\Psi_{j}\right\rangle+\frac{y_{j}}{y_{r}-y_{j}}\left|\Psi_{r}\right\rangle\right) \\
= & (1+G) \sum_{j=1}^{M}\left(y_{j}|\Psi\rangle-Q^{+}\left|\Psi_{j}\right\rangle\right)-2 G Q^{+} \sum_{j=1}^{M}\left(\frac{z_{1}^{2} s}{y_{j}-z_{1}^{2}}+\frac{z_{2}^{2} s}{y_{j}-z_{2}^{2}}\right)\left|\Psi_{j}\right\rangle \\
& +2 G Q^{+} \sum_{j=1}^{M} \sum_{r \neq j}^{M} \frac{y_{r}}{y_{j}-y_{r}}\left|\Psi_{j}\right\rangle \\
= & (1+G) \sum_{j=1}^{M}\left(y_{j}|\Psi\rangle-Q^{+}\left|\Psi_{j}\right\rangle\right)-2 G Q^{+} \sum_{j=1}^{M}\left(\frac{z_{1}^{2} s}{y_{j}-z_{1}^{2}}+\frac{z_{2}^{2} s}{y_{j}-z_{2}^{2}}\right)\left|\Psi_{j}\right\rangle \\
& +2 G Q^{+} \sum_{j=1}^{M} \sum_{r \neq j}^{M} \frac{y_{r}}{y_{j}-y_{r}}\left|\Psi_{j}\right\rangle .
\end{aligned}
$$

The terms proportional to $\left|\Psi_{j}\right\rangle$ cancel provided

$$
(1+G)+2 G\left(\frac{z_{1}^{2} s}{y_{j}-z_{1}^{2}}+\frac{z_{2}^{2} s}{y_{j}-z_{2}^{2}}\right)=2 G \sum_{r \neq j}^{M} \frac{y_{r}}{y_{j}-y_{r}}, \quad k=1, \ldots, M
$$

which can be equivalently written as

$$
\frac{G^{-1}+2 M+4 s-1}{y_{k}}+\frac{2 s}{y_{k}-z_{1}^{2}}+\frac{2 s}{y_{k}-z_{2}^{2}}=\sum_{j \neq k}^{M} \frac{2}{y_{k}-y_{j}}, \quad k=1, \ldots, M .
$$

For each solution of the coupled equations (11), $|\Psi\rangle$ is an eigenstate of (2) with energy eigenvalue given by

$$
E=(1+G) \sum_{k=1}^{M} y_{k}
$$

\subsection{Duality relations}

For $z_{2} \neq 0$, from the commutation relations (1) the following equation can be shown to hold using proof by induction:

$$
\left[H,(C(0))^{M}\right]=-M Q^{+}(C(0))^{M-1}\left(1+G M+2 G S^{z}\right) .
$$

If a state $|\psi\rangle$ has spin $m$ (the eigenvalue of $S^{z}$ ) the above equation shows that choosing

$$
1+G M+2 G m=0
$$

so $M=-2 m-G^{-1}$, the two states $|\psi\rangle$ and $|\tilde{\psi}\rangle=(C(0))^{M}|\psi\rangle$ have the same energy. We call the states $|\psi\rangle$ and $|\tilde{\psi}\rangle$ dual states. Since the state $|\psi\rangle$ has spin $m$, the state $|\tilde{\psi}\rangle$ has spin $m+M=-m-G^{-1}$. We identify the following special cases: 


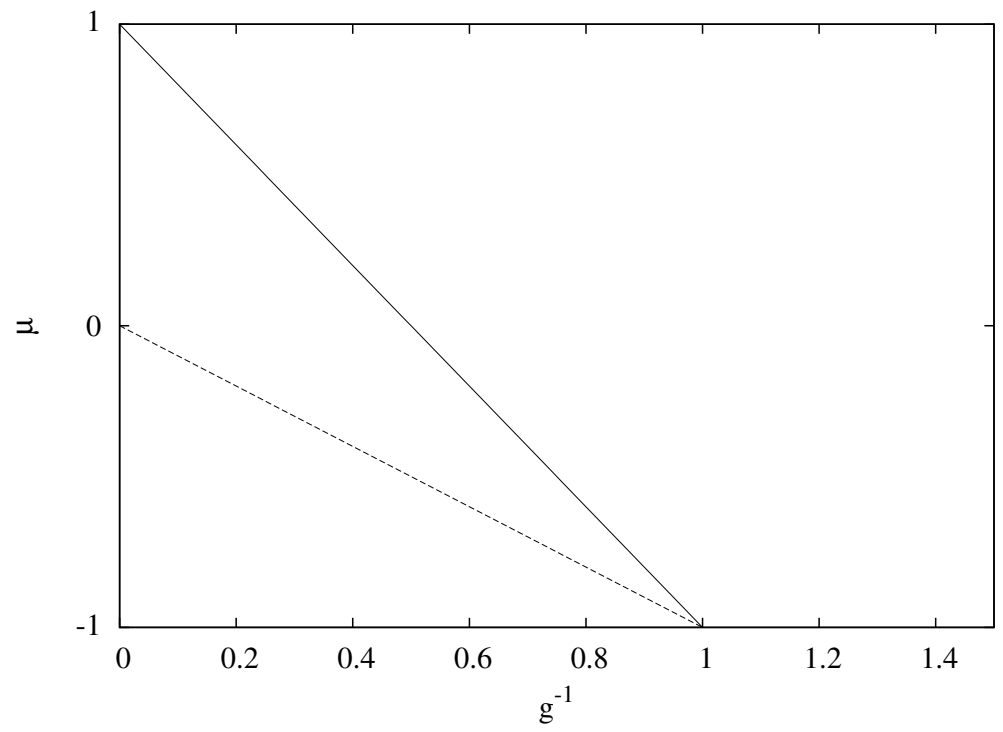

Figure 1. Duality diagram. The solid line represents equation (7), on which the intensive ground-state energy is zero. The dotted line represents equation (9). The mean-field analysis predicts that there are gapless excitations on equation (9) as $z_{2} \rightarrow 0$, but also that the limits $\mu \rightarrow-g^{-1}$ and $z_{2} \rightarrow 0$ do not commute in equation (5).

- The dual of the lowest weight state $|\psi\rangle=|s,-s\rangle \otimes|s,-s\rangle$ occurs when $M=4 s-G^{-1}$ leading to $|\tilde{\psi}\rangle$ having spin $m=2 s-G^{-1}$. In the classical limit $s \rightarrow \infty$ this yields equation (7), for which the ground-state energy is zero. Explicitly

$$
|\tilde{\psi}\rangle=(C(0))^{M}|s,-s\rangle \otimes|s,-s\rangle .
$$

- If $M=0$ then a state is self-dual when $m=-(2 G)^{-1}$. In the classical limit $s \rightarrow \infty$ this yields equation (9).

Note that neither line given by (7) nor (9) depends on the variables $z_{1}$ and $z_{2}$. However, as already seen in the mean-field analysis, caution must be taken when considering the limit $z_{2} \rightarrow 0$. Since $C(0)$ is singular in the limit $z_{2} \rightarrow 0$, we define rescaled operators

$$
\tilde{C}(0)=z_{1} z_{2} C(0)=z_{2} S_{1}^{+}+z_{1} S_{2}^{+} .
$$

Equation (12) assumes the form

$$
\left[H,(\tilde{C}(0))^{M}\right]=-z_{1} z_{2} M Q^{+}(\tilde{C}(0))^{M-1}\left(1+G M+2 G S^{z}\right) .
$$

It is easily recognized that the commutator vanishes in the limit $z_{2} \rightarrow 0$. In this case the classes of states with the same energy generally have greater cardinality than those of the dual state classes identified above for $z_{2} \neq 0$.

\subsection{Classical limit of the ground-state exact solution}

Following $[4,11,16]$, we look to solve the Bethe ansatz equations in the classical limit under the assumption that in this limit the roots of (11) are densely distributed on a curve $\Gamma$ in the complex plane which is invariant under reflection about the real axis. Introducing $r(y)$ as the density function on $\Gamma$ for the roots, we divide (11) by $2 s$ and take the limit $s \rightarrow \infty$ to obtain

$$
\frac{2 g^{-1}+2 \mu}{y}+\frac{1}{y-z_{1}^{2}}+\frac{1}{y-z_{2}^{2}}=2 P \int_{\Gamma}\left|d y^{\prime}\right| \frac{r\left(y^{\prime}\right)}{y-y^{\prime}},
$$


where $P$ refers to the Cauchy principal value for the integral. To solve the problem we define an analytic function $h\left(y^{\prime}\right)$ outside $\Gamma$ such that

$$
r\left(y^{\prime}\right)=h_{+}\left(y^{\prime}\right)-h_{-}\left(y^{\prime}\right)
$$

where $h_{+}\left(y^{\prime}\right)$ and $h_{-}\left(y^{\prime}\right)$ are the limiting values of $h\left(y^{\prime}\right)$ to the right and left of $\Gamma$. We can then rewrite $(15)$ as

$$
\frac{2 g^{-1}+2 \mu}{y}+\frac{1}{y-z_{1}^{2}}+\frac{1}{y-z_{2}^{2}}=2 \oint_{L} d y^{\prime} \frac{h\left(y^{\prime}\right)}{y-y^{\prime}},
$$

where $L$ encircles $\Gamma$. Next we take the following ansatz:

$$
h(y)=\frac{R(y)}{2 \pi i}\left[\frac{\phi\left(z_{1}\right)}{z_{1}^{2}-y}+\frac{\phi\left(z_{2}\right)}{z_{2}^{2}-y}+\frac{S}{y}\right]
$$

where

$$
R(y)=\sqrt{(y-a)(y-b)}
$$

with $a, b=a^{*}$ denoting the end points of the $\operatorname{arc} \Gamma$. Substituting (17) into (16) and evaluating the contour integral by residues leads to the conditions

$$
\phi\left(z_{j}\right)=\frac{1}{2 \sqrt{\left(z_{j}^{2}-a\right)\left(z_{j}^{2}-b\right)}}, \quad S=\phi\left(z_{1}\right)+\phi\left(z_{2}\right), \quad S=\frac{g^{-1}+\mu}{\sqrt{a b}} .
$$

These combine to give

$$
\frac{2}{g}+2 \mu=\frac{\sqrt{a b}}{\sqrt{\left(z_{1}^{2}-a\right)\left(z_{1}^{2}-b\right)}}+\frac{\sqrt{a b}}{\sqrt{\left(z_{2}^{2}-a\right)\left(z_{2}^{2}-b\right)}} .
$$

We may also compute

$$
\begin{aligned}
\mu & =-1+\int_{\Gamma}\left|d y^{\prime}\right| r\left(y^{\prime}\right)=-1+\oint_{L} d y^{\prime} h\left(y^{\prime}\right) \\
& =\left(\frac{a+b}{2}\right) S-z_{1}^{2} \phi\left(z_{1}\right)-z_{2}^{2} \phi\left(z_{2}\right)-\frac{(\sqrt{a}-\sqrt{b})^{2}}{2} S \\
\Longrightarrow & \frac{2}{g}=\frac{z_{1}^{2}}{\sqrt{\left(z_{1}^{2}-a\right)\left(z_{1}^{2}-b\right)}}+\frac{z_{2}^{2}}{\sqrt{\left(z_{2}^{2}-a\right)\left(z_{2}^{2}-b\right)}} .
\end{aligned}
$$

Identifying $\rho=\sqrt{a b}$ and $\Delta^{2}=a+b+2 \sqrt{a b}$, equations (18), (19) are precisely the mean-field equations (5), (4) respectively. This would suggest that the mean-field equations should be exact in the classical limit as they are reproducible from the exact Bethe ansatz solution. However, the above calculations assume that in the classical limit the roots of the Bethe ansatz equations become dense on a curve $\Gamma$. This assumption is not always valid. Suppose that the curve $\Gamma$ closes such that the end points $a, b$ become real and equal. Then equations (18), (19) reduce to

$$
\frac{2}{g}+2 \mu=\frac{|a|}{\left|z_{1}^{2}-a\right|}+\frac{|a|}{\left|z_{2}^{2}-a\right|}, \quad \frac{2}{g}=\frac{z_{1}^{2}}{\left|z_{1}^{2}-a\right|}+\frac{z_{2}^{2}}{\left|z_{2}^{2}-a\right|} .
$$

If $a \geq 0$ the only solution is $\mu=-1$, whereas if $a<0$ we obtain equation (7). But the groundstate roots in this instance are all zero, as the ground state is dual to the lowest weight state as shown in Subsection 3.1 (see equation (13)). Thus taking $s \rightarrow \infty$ after $g \rightarrow 2(1-\mu)^{-1}$ leads 
to a delta function distribution of roots at the origin, while taking $g \rightarrow 2(1-\mu)^{-1}$ from below after $s \rightarrow \infty$ leads to the roots being distributed on a closed curve enclosing the origin. We may identify equation (7) as the boundary at which mean-field results break down. In the region immediately beyond the line given by equation (7), we cannot assume that the ground-state roots of the Bethe ansatz equations are densely distributed on a single curve in order to produce the mean-field equations from the Bethe ansatz solution. A numerical study of this case has been undertaken in [11].

\subsection{The limiting case $z_{2}=0$}

In view of equation (8), which indicates that the minimum excitation energy approaches zero as $z_{2} \rightarrow 0$, here we set $z_{2}=0$ from the outset. We also fix the energy scale by letting $z_{1}=1$, in which case the Hamiltonian is simply

$$
H=(1+G)\left(S_{1}^{z}+s\right)-G S_{1}^{+} S_{1}^{-} .
$$

The above Hamiltonian is diagonal in the basis (3) with eigenvalues

$$
E=m_{1}+s-G\left(s^{2}-m_{1}^{2}\right) .
$$

For each value of $m=m_{1}+m_{2}$ we can determine the ground state energy, and the gap to the first excited state, by considering where level crossings occur. We need to consider several different cases. Starting with $m=m_{1}+m_{2}<0$ we have

$$
\begin{aligned}
& m_{1}=-s, \quad m_{2}=m+s \quad \Longrightarrow \quad E=0, \\
& m_{1}=-s+1, \quad m_{2}=m+s-1 \quad \Longrightarrow \quad E=(1+G)-2 s G .
\end{aligned}
$$

In the classical limit it is seen that that for $m<0$ a first level crossing occurs at

$$
g=2 .
$$

Next consider

$$
\begin{aligned}
& m_{1}=s+m-1, \quad m_{2}=-s+1 \quad \Longrightarrow \quad E=2 s+m-1+G\left((m-1)^{2}+2 s(m-1)\right), \\
& m_{1}=s+m, \quad m_{2}=-s \quad \Longrightarrow \quad E=2 s+m+G\left(m^{2}+2 s m\right) .
\end{aligned}
$$

Here there is an energy gap

$$
\Delta E=|1+G(2 m-1+2 s)| .
$$

In the classical limit the final level crossing occurs when

$$
\mu=-\frac{1}{2}-\frac{1}{g},
$$

which requires that $\mu<-1 / 2$.

Now we consider the case for $m \geq 0$ :

$$
\begin{aligned}
& m_{1}=-s+m, \quad m_{2}=s \quad \Longrightarrow \quad E=m+G\left(m^{2}-2 s m\right), \\
& m_{1}=-s+m+1, \quad m_{2}=s-1 \quad \Longrightarrow \quad E=m+1+G\left((m+1)^{2}-2 s(m+1)\right) .
\end{aligned}
$$

The gap

$$
\Delta E=|1+G(2 m+1-2 s)|
$$


goes to zero in the classical limit when

$$
\mu=\frac{1}{2}-\frac{1}{g} \text {. }
$$

This requires that $0 \leq \mu<1 / 2$. In the region bounded by the equations (21), (22), (23) the level crossings become dense in the classical limit, giving rise to a gapless phase. The following relations hold for the gap between the ground state and first excited state in the regions depicted in the phase diagram of Fig. 2:

$$
\begin{array}{ll}
\text { I : } & \Delta E=1+g(\mu-1 / 2), \\
\text { II : } & \Delta E=1-g / 2, \\
\text { III : } & \Delta E=-1-g(\mu+1 / 2), \\
\text { IV : } & \Delta E=0 .
\end{array}
$$

Moreover, when $z_{2}=0$ the Hamiltonian (20) acquires an $\mathfrak{s u}(2)$ symmetry due to commutativity with the generators $\left\{S_{2}^{z}, S_{2}^{+}, S_{2}^{-}\right\}$. In view of this, we define an order parameter

$$
\mathcal{O}=\lim _{s \rightarrow \infty} \frac{\left\langle S_{2}^{z}\right\rangle}{s} .
$$

Analogous to the procedure described above, the following relations are found for the order parameter in the regions depicted in the phase diagram of Fig. 2:

$$
\begin{array}{ll}
\text { I : } & \mathcal{O}=1 \\
\text { II : } & \mathcal{O}=1+2 \mu \\
\text { III : } & \mathcal{O}=-1 \\
\text { IV : } & \mathcal{O}=2\left(\mu+g^{-1}\right) .
\end{array}
$$

Only in the gapless region IV does the order parameter vary as a function of $g$. The boundary lines between the regions are associated with discontinuities in the partial derivatives of $\mathcal{O}$.

There is clear discrepancy between Figs. 1 and 2 with respect to the vanishing of the energy gap. In deriving the two diagrams we consider behaviours as $G \rightarrow 0, s \rightarrow \infty, m \rightarrow \infty$ with $g=4 s G, \mu=m(2 s)^{-1}$, and as $z_{2} \rightarrow 0$, yielding phase boundary lines as functions of the coupling parameter $g$. In the next subsection we provide calculations which support the phase diagram shown in Fig. 2.

\subsection{The limiting case $\mu=-1$}

In the sector where $\mu=-1+(2 s)^{-1}$, or equivalently $m=1-2 s$, the Hilbert space is twodimensional and exact results are easily extracted. In matrix form, with respect to the basis

$$
\{|s,-s+1\rangle \otimes|s,-s\rangle,|s,-s\rangle \otimes|s,-s+1\rangle\}
$$

the Hamiltonian is

$$
H=\left(\begin{array}{cc}
(1+G-2 s G) z_{1}^{2} & -2 s G z_{1} z_{2} \\
-2 s G z_{1} z_{2} & (1+G-2 s G) z_{2}^{2}
\end{array}\right)
$$

with the ground-state energy

$$
E=\frac{\left(1-g / 2+g(4 s)^{-1}\right)\left(z_{1}^{2}+z_{2}^{2}\right)}{2}-\frac{1}{2} \sqrt{\left(1-g / 2+g(4 s)^{-1}\right)^{2}\left(z_{1}^{2}-z_{2}^{2}\right)^{2}+g^{2} z_{1}^{2} z_{2}^{2}}
$$




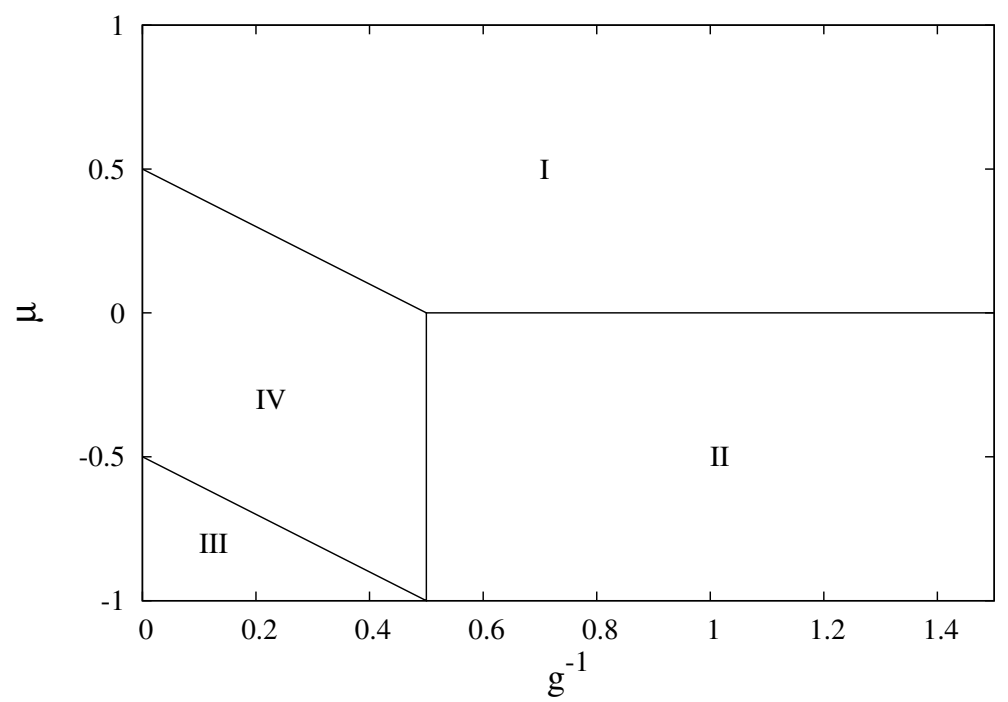

Figure 2. Order parameter phase diagram. Expressions for the gap between the ground state and first excited state for $z_{2}=0$ in the classical limit are given by equations (24)-(27), and expressions for the order parameter (28) are given by equations (29)-(32). Region IV is gapless, and is the only region in which the order parameter varies as a function of $g$. Note that the boundary lines between regions I and IV, and also III and IV, are given by (10) for $\rho>0$ and $\rho<0$ respectively. Neither lines given by equation (7) nor equation (9) of Fig. 1 are present in the diagram.

and energy gap to the excited state

$$
\Delta E=\sqrt{\left(1-g / 2+g(4 s)^{-1}\right)^{2}\left(z_{1}^{2}-z_{2}^{2}\right)^{2}+g^{2} z_{1}^{2} z_{2}^{2}} .
$$

The mean-field results of Section 2 indicate that the vanishing of the energy gap depends on how the limits $z_{2} \rightarrow 0$ and $\rho \rightarrow 0$ are taken. It is found from (33) that

$$
\lim _{g \rightarrow 2} \lim _{z_{2} \rightarrow 0} \lim _{s \rightarrow \infty} \Delta E=0
$$

with the result being invariant with respect to interchanging the order of the limits. Moreover, as $s \rightarrow \infty$ the only solution for $\Delta E=0$ when $z_{1} \neq 0$ is given by $z_{2}=0, g=2$, meaning that gapless excitations do not occur on the line given by equation (9) in the limit $\mu \rightarrow-1$.

Next consider

$$
\frac{\partial(\Delta E)}{\partial g}=\frac{g\left(z_{1}^{2}+z_{2}^{2}\right)^{2}+\left(-2+(1-g) s^{-1}+g\left(4 s^{2}\right)^{-1}\right)\left(z_{1}^{2}-z_{2}^{2}\right)^{2}}{4 \Delta E} .
$$

We then have

$$
\begin{aligned}
\lim _{s \rightarrow \infty} & \lim _{z_{2} \rightarrow 0} \lim _{g \rightarrow 2} \frac{\partial(\Delta E)}{\partial g} \\
& =\lim _{s \rightarrow \infty} \lim _{z_{2} \rightarrow 0} \lim _{g \rightarrow 2} \frac{g\left(z_{1}^{2}+z_{2}^{2}\right)^{2}+\left(-2+(1-g) s^{-1}+g\left(4 s^{2}\right)^{-1}\right)\left(z_{1}^{2}-z_{2}^{2}\right)^{2}}{4 \sqrt{\left(1-g / 2+g(4 s)^{-1}\right)^{2}\left(z_{1}^{2}-z_{2}^{2}\right)^{2}+g^{2} z_{1}^{2} z_{2}^{2}}} \\
& =\lim _{s \rightarrow \infty} \lim _{z_{2} \rightarrow 0} \frac{2\left(z_{1}^{2}+z_{2}^{2}\right)^{2}+\left(-2-s^{-1}+\left(2 s^{2}\right)^{-1}\right)\left(z_{1}^{2}-z_{2}^{2}\right)^{2}}{4 \sqrt{\left((2 s)^{-2}\left(z_{1}^{2}-z_{2}^{2}\right)^{2}+4 z_{1}^{2} z_{2}^{2}\right.}} \\
& =\lim _{s \rightarrow \infty} \frac{2 z_{1}^{4}+\left(-2-s^{-1}+\left(2 s^{2}\right)^{-1}\right) z_{1}^{4}}{2 s^{-1} z_{1}^{2}}=-\frac{z_{1}^{2}}{2} .
\end{aligned}
$$


On the other hand

$$
\begin{aligned}
\lim _{z_{2} \rightarrow 0} \lim _{g \rightarrow 2} \lim _{s \rightarrow \infty} \frac{\partial(\Delta E)}{\partial g} & =\lim _{z_{2} \rightarrow 0} \lim _{g \rightarrow 2} \lim _{s \rightarrow \infty} \frac{g\left(z_{1}^{2}+z_{2}^{2}\right)^{2}-\left(2-\left(1-g+g(4 s)^{-1}\right) s^{-1}\right)\left(z_{1}^{2}-z_{2}^{2}\right)^{2}}{4 \sqrt{\left(1+g(4 s)^{-1}-g / 2\right)^{2}\left(z_{1}^{2}-z_{2}^{2}\right)^{2}+g^{2} z_{1}^{2} z_{2}^{2}}} \\
& =\lim _{z_{2} \rightarrow 0} \lim _{g \rightarrow 2} \frac{g\left(z_{1}^{2}+z_{2}^{2}\right)^{2}-2\left(z_{1}^{2}-z_{2}^{2}\right)^{2}}{4 \sqrt{(1-g / 2)^{2}\left(z_{1}^{2}-z_{2}^{2}\right)^{2}+g^{2} z_{1}^{2} z_{2}^{2}}} \\
& =\lim _{z_{2} \rightarrow 0} \frac{2\left(z_{1}^{2}+z_{2}^{2}\right)^{2}-2\left(z_{1}^{2}-z_{2}^{2}\right)^{2}}{4 \sqrt{4 z_{1}^{2} z_{2}^{2}}}=\lim _{z_{2} \rightarrow 0} z_{1} z_{2}=0 .
\end{aligned}
$$

This shows that the non-commutativity of limits is not just an artefact of the mean-field approximation, but is an intrinsic property of the model. Similar calculations also show

$$
\begin{array}{ll}
\lim _{s \rightarrow \infty} \lim _{g \rightarrow 2} \lim _{z_{2} \rightarrow 0} \frac{\partial(\Delta E)}{\partial g}=-\frac{z_{1}^{2}}{2}, & \lim _{z_{2} \rightarrow 0} \lim _{s \rightarrow \infty} \lim _{g \rightarrow 2} \frac{\partial(\Delta E)}{\partial g}=0, \\
\lim _{g \rightarrow 2^{+}} \lim _{z_{2} \rightarrow 0} \lim _{s \rightarrow \infty} \frac{\partial(\Delta E)}{\partial g}=\frac{z_{1}^{2}}{2}, & \lim _{g \rightarrow 2^{-}} \lim _{z_{2} \rightarrow 0} \lim _{s \rightarrow \infty} \frac{\partial(\Delta E)}{\partial g}=-\frac{z_{1}^{2}}{2}, \\
\lim _{g \rightarrow 2^{+}} \lim _{s \rightarrow \infty} \lim _{z_{2} \rightarrow 0} \frac{\partial(\Delta E)}{\partial g}=\frac{z_{1}^{2}}{2}, & \lim _{g \rightarrow 2^{-}} \lim _{s \rightarrow \infty} \lim _{z_{2} \rightarrow 0} \frac{\partial(\Delta E)}{\partial g}=-\frac{z_{1}^{2}}{2} .
\end{array}
$$

\section{Indicators of quantum phase transitions}

In the remaining subsections we compute various quantities which accord with the phase diagram shown in Fig. 2. While there have been numerous studies of quantum phase transitions, there still lacks a definitive property which may be used to identify them. Using numerical diagonalisation of the Hamiltonian (2), below we compute the energy gap, fidelity, and entanglement (as given by the von Neumann entropy). Each quantity is plotted as a function of the parameter $g^{-1}$. We fix $\mu=-0.99, z_{1}=1$, and adopt the parameterisation $z_{2}=(2000 s)^{-1 / 2}$ such that $z_{2} \rightarrow 0$ as $s \rightarrow \infty$. We focus on this case as it permits us to compactly cover the regions II, III and IV in Fig. 2.

\subsection{Energy gap}

In Fig. 3 we plot the energy gap. There are two different points of minima corresponding to the boundary lines between region III and IV, and between region IV and II, in Fig. 2. It is seen in the inset that as $s$ increases the energy gap decreases in region IV of Fig. 2. The results appear more consistent with Fig. 2 rather than Fig. 1, as to be expected in view of the results from Subsection 3.4.

\subsection{Fidelity}

Another means to investigate quantum phase transitions is fidelity [21, 22], a concept widely used in quantum information theory. The fidelity of two states is simply defined as the modulus of the inner product

$$
\mathcal{F}(\psi, \phi)=|\langle\psi \mid \phi\rangle|
$$

Computing this quantity for ground-state vectors associated with Hamiltonians differing only by a small change in the coupling parameter, the fidelity may be sensitive to variations of the coupling parameter. Generally a minimum in the fidelity points to a rapid change in the ground-state properties, which may indicate a critical point of the system. 


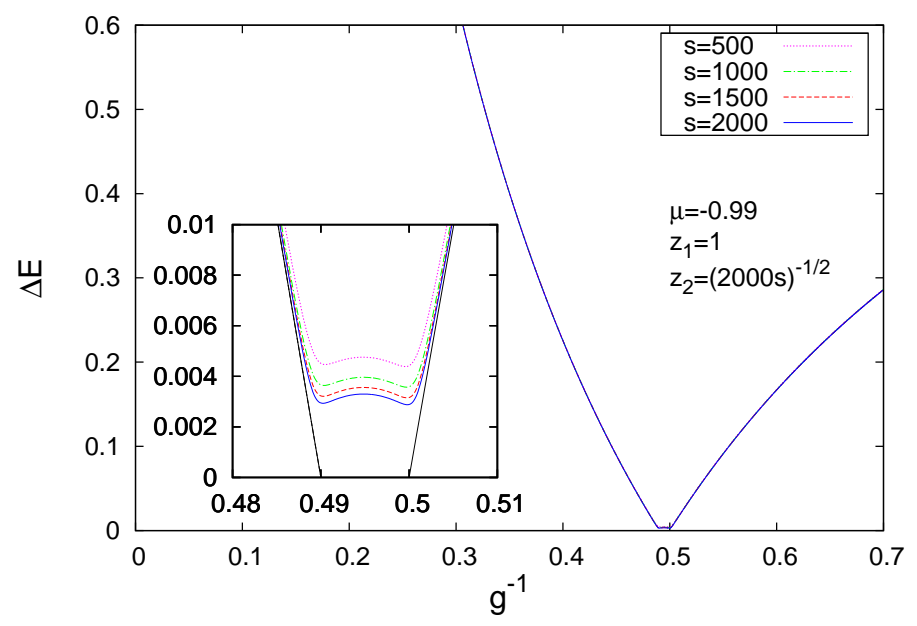

Figure 3. Energy gap between the ground state and the first excited state as a function of $g^{-1}$, with $\mu=$ -0.99 . The results shown in the inset indicate the tendency towards vanishing gap in region IV as $s \rightarrow \infty$. The solid black line represents the analytic curve for the energy gap as given by equations (25), (26) for when $z_{2}=0$.

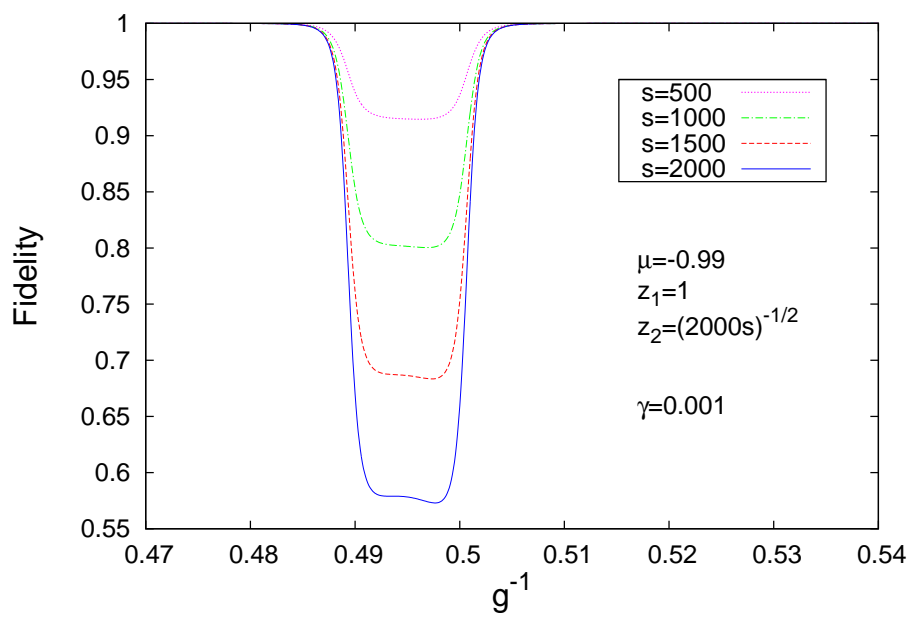

Figure 4. Ground-state fidelity as a function of $g^{-1}$, with $\mu=-0.99$ and $\gamma=0.001$ for different values of $s$ as indicated in the legend. The results show significantly decreasing fidelity in region IV as $s \rightarrow \infty$.

Here we take two coupling values

$$
\left(g^{ \pm}\right)^{-1}=g^{-1}(1 \pm \gamma)
$$

with $\gamma=0.001$ to compute the fidelity of the ground states associated with $g^{ \pm}$, and then consider the behaviour of the fidelity as $g$ is varied. As is seen in Fig. 4, for values of the parameter $g^{-1}$ above 0.5 and values below 0.49 , the fidelity is close to 1 , but suffers an abrupt change at these two points. The two points correspond to the boundary lines between regions II and IV and regions IV and III for $\mu=-0.99$ in the phase diagram Fig. 2. Also, it can be seen that the minimum of the fidelity is decreasing as $s$ increases.

\subsection{Entanglement}

The coupled-spin model is naturally seen as a bipartite system. In this case we can use the von Neumann entropy of the reduced density operator to quantify the entanglement of the 


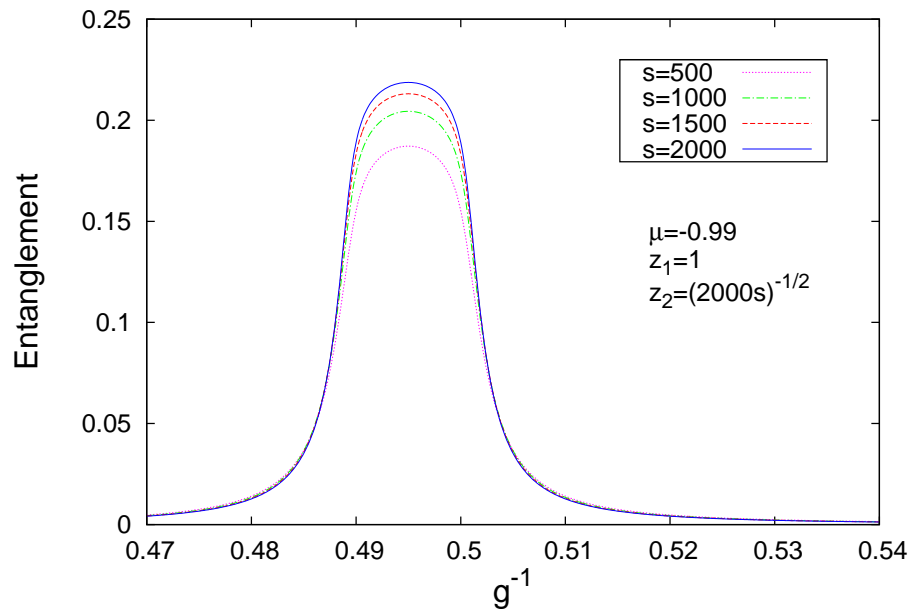

Figure 5. Ground-state entanglement as function of $g^{-1}$ for $\mu=-0.99$ for different values of $s$ as indicated in the legend. The results show significantly increasing entanglement in region IV as $s \rightarrow \infty$.

system $[6,12,14,17]$. Using the fact that the total spin $m$ is conserved, we can write a general state of the system (for $m \leq 0$ ) in terms of (3) by

$$
|\Psi\rangle=\sum_{j=-s}^{m+s} d_{j}|s, j\rangle \otimes|s, m-j\rangle
$$

for some complex numbers $d_{j}$. The density operator for the state (34) is given by

$$
\rho=|\Psi\rangle\left\langle\Psi\left|=\sum_{i, j=-s}^{m+s} d_{i} d_{j}^{*}\right| s, i\right\rangle\langle s, j|\otimes| s, m-i\rangle\langle s, m-j| .
$$

We take the partial trace with respect to the spin sector 2, yielding the reduced density operator for the spin sector 1:

$$
\rho_{1}=\operatorname{tr}_{2}(\rho)=\sum_{j=-s}^{m+s}\left|d_{j}\right|^{2}|s, j\rangle\langle s, j|
$$

The von Neumann entropy of entanglement for the state is given by

$$
\mathcal{E}\left(\rho_{1}\right)=-\operatorname{tr}\left[\rho_{1} \log _{2}\left(\rho_{1}\right)\right]=-\sum_{j=-s}^{m+s}\left|d_{j}\right|^{2} \log _{2}\left(\left|d_{j}\right|^{2}\right) .
$$

Using the formula (35) we compute the entanglement of the ground state with $\mu=-0.99$ as function of $g^{-1}$, as shown in Fig. 5. We can identify two abrupt changes in the entanglement value about the points $g^{-1}=0.49$ and $g^{-1}=0.5$ in agreement with energy gap and fidelity analysis. Note that the entanglement variation gets sharper as the value of $s$ is increased.

\section{Conclusion}

A main outcome of this study is that the limiting behaviours of the Hamiltonian (2) depend on the order in which limits are taken. This was first observed through the mean-field analysis conducted in Section 2. In the following Section 3, concerned with exact techniques, two phase 
diagrams were obtained as depicted in Figs. 1 and 2. Subsequent exact calculations, undertaken in Subsection 3.4, gave weight to the phase diagram of Fig. 2. This was further supported by numerical calculation of the energy gap, fidelity, and entanglement, conducted in Section 4. The discrepancy between the two diagrams has its origin in assumptions regarding limits commuting, particularly those involving $z_{2} \rightarrow 0$. In part, this can be traced back to the vanishing of the commutator (14) as $z_{2} \rightarrow 0$. The calculations of the partial derivative of the energy gap with respect to the coupling $g$ given in Subsection 3.4 provided concrete examples of noncommutativity of limits.

The model studied here may be viewed as an analogue of the $p+i p$ pairing Hamiltonian. Letting $c_{\mathbf{k}}, c_{\mathbf{k}}^{\dagger}$ denote annihilation and creation operators for two-dimensional spinless or spinpolarised fermions of mass $m$ with momentum $\mathbf{k}=\left(k_{x}, k_{y}\right)$, the Hamiltonian reads $[4,7,16]$

$$
H=\sum_{\mathbf{k}} \frac{|\mathbf{k}|^{2}}{2 m} c_{\mathbf{k}}^{\dagger} c_{\mathbf{k}}-\frac{G}{4 m} \sum_{\mathbf{k} \neq \mathbf{k}^{\prime}}\left(k_{x}+i k_{y}\right)\left(k_{x}^{\prime}-i k_{y}^{\prime}\right) c_{\mathbf{k}}^{\dagger} c_{-\mathbf{k}}^{\dagger} c_{-\mathbf{k}^{\prime}} c_{\mathbf{k}^{\prime}},
$$

where $G$ is the coupling constant. The Hamiltonian commutes with the total fermion number

$$
N=\sum_{\mathbf{k}} c_{\mathbf{k}}^{\dagger} c_{\mathbf{k}}
$$

This model has attracted interest due to it topological properties $[1,3,10,15,18,20]$. The phase diagram of the model is characterized by two lines known as the Moore-Read line and the Read-Green line. Letting $L$ denote the number of momenta $\mathbf{k}$, the Moore-Read line is given by $G^{-1}=L-N / 2$, for which the ground state energy is zero. The Read-Green line is given by $G^{-1}=(L-N / 2) / 2$, which was shown in [16] to correspond to a third-order quantum phase transition. Equation (7) is the analogue of the Moore-Read line for the coupled spin Hamiltonian (2), while equation (9) is the analogue of the Read-Green line. The phase diagram for the $p+i p$ pairing Hamiltonian has the same qualitative features as Fig. 1. The topological nature of this model is reflected by the fact that the equations for the Moore-Read and ReadGreen lines are independent of the distribution of the momenta $\mathbf{k}$, so the phase diagram is robust against changes to the momentum distribution. Analogously, equations (7) and (9) do not depend on the variables $z_{1}$ and $z_{2}$ for the coupled-spin model, which may be viewed as a degenerate limiting case of the $p+i p$ pairing Hamiltonian as discussed in [11]. Our studies indicate that new features of the phase diagram appear in the limit $z_{2} \rightarrow 0$. This observation prompts a closer examination of the $p+i p$ model in the limit when some of the momenta go to zero, particularly with respect to the commutivity or otherwise of particular limits. This aspect is significant because the occurrence of zero momenta in the $p+i p$ model is intimately related to the existence of Majorana fermions, exhibiting non-abelian anyonic statistics, a topic which has been studied in $[2,5,8,15,19]$.

\section{Acknowledgements}

EM is supported by CAPES (Brazil). JL is supported by the Australian Research Council through Discovery Project DP110101414. We thank the anonymous referees for their recommendations on revising the manuscript. JL thanks Germán Sierra for numerous insightful discussions.

\section{References}

[1] Bray-Ali N., Ding L., Haas S., Topological order in paired states of fermions in two dimensions with breaking of parity and time-reversal symmetries, Phys. Rev. B 80 (2009), 180504(R), 4 pages, arXiv:0905.2946. 
[2] Cheng M., Lutchyn R.M., Das Sarma S., Topological protection of Majorana qubits, Phys. Rev. B 85 (2012), 165124, 8 pages, arXiv:1112.3662.

[3] Dubail J., Read N., Entanglement spectra of complex paired superfluids, Phys. Rev. Lett. 107 (2011), 157001, 5 pages, arXiv:1105.4808.

[4] Dunning C., Ibañez M., Links J., Sierra G., Zhao S.-Y., Exact solution of the $p+i p$ pairing Hamiltonian and a hierarchy of integrable models, J. Stat. Mech. Theory Exp. 2010 (2010), P08025, 62 pages, arXiv:1001.1591.

[5] Gurarie V., Radzihovsky L., Zero modes of two-dimensional chiral p-wave superconductors, Phys. Rev. B 75 (2007), 212509, 4 pages, cond-mat/0610094.

[6] Hines A.P., McKenzie R.H., Milburn G.J., Entanglement of two-mode Bose-Einstein condensates, Phys. Rev. A 67 (2003), 013609, 10 pages, quant-ph/0209122.

[7] Ibañez M., Links J., Sierra G., Zhao S.-Y., Exactly solvable pairing model for superconductors with $p_{x}+i p_{y^{-}}$ wave symmetry, Phys. Rev. B 79 (2009), 180501, 4 pages, arXiv:0810.0340.

[8] Kraus Y.E., Auerbach A., Fertig H.A., Simon S.H., Majorana fermions of a two-dimensional $p_{x}+i p_{y}$ superconductor, Phys. Rev. B $\mathbf{7 9}$ (2009), 134515, 15 pages, arXiv:0904.2920.

[9] Lerma H. S., Dukelsky J., The Lipkin-Meshkov-Glick model as a particular limit of the SU(1,1) RichardsonGaudin integrable models, Nuclear Phys. B 870 (2013), 421-443, arXiv:1212.3238.

[10] Levinsen J., Cooper N.R., Shlyapnikov G.V., Topological $p_{x}+i p_{y}$ superfluid phase of fermionic polar molecules, Phys. Rev. A 84 (2011), 013603, 13 pages, arXiv:1103.3859.

[11] Marquette I., Links J., Generalised Heine-Stieltjes and Van Vleck polynomials associated with degenerate, integrable BCS models, J. Stat. Mech. Theory Exp. 2012 (2012), P08019, 22 pages, arXiv:1206.2988.

[12] Osborne T.J., Nielsen M.A., Entanglement in a simple quantum phase transition, Phys. Rev. A 66 (2002), 032110, 14 pages, quant-ph/0202162.

[13] Osterloh A., Amico L., Falci G., Fazio R., Scaling of entanglement close to a quantum phase transition, Nature 416 (2002), 608-610, quant-ph/0202029.

[14] Pan F., Draayer J.P., Quantum critical behavior of two coupled Bose-Einstein condensates, Phys. Lett. A 339 (2005), 403-407, cond-mat/0410423.

[15] Read N., Green D., Paired states of fermions in two dimensions with breaking of parity and time-reversal symmetries and the fractional quantum Hall effect, Phys. Rev. B 61 (2000), 10267-10297, cond-mat/9906453.

[16] Rombouts S.M.A., Dukelsky J., Ortiz G., Quantum phase diagram of the integrable $p_{x}+i p_{y}$ fermionic superfluid, Phys. Rev. B $\mathbf{8 2}$ (2010), 224510, 19 pages, arXiv:1008.3406.

[17] Rubeni D., Foerster A., Mattei E., Roditi I., Quantum phase transitions in Bose-Einstein condensates from a Bethe ansatz perspective, Nuclear Phys. B 856 (2012), 698-715, arXiv:1204.5790.

[18] Stone M., Chung S.B., Fusion rules asnd vortices in $p_{x}+i p_{y}$ superconductors, Phys. Rev. B 73 (2006), 014505, 11 pages, cond-mat/0505515.

[19] Tewari S., Das Sarma S., Nayak C., Zhang C.W., Zoller P., Quantum computation using vortices and Majorana zero models of a $p_{x}+i p_{y}$ superfluid of fermionic cold atoms, Phys. Rev. Lett. 98 (2007), 010506, 4 pages, quant-ph/0606101.

[20] Volovik G.E., Analog of quantum Hall effect in supefluid ${ }^{3}$ He film, Sov. Phys. JETP 67 (1988), $1804-1811$.

[21] Zanardi P., Paunković N., Ground state overlap and quantum phase transitions, Phys. Rev. E 74 (2006), 031123, 6 pages, quant-ph/0512249.

[22] Zhou H.-Q., Barjaktarevič J.P., Fidelity and quantum phase transitions, J. Phys. A: Math. Theor. 41 (2008), 412001, 7 pages, cond-mat/0701608. 